\title{
Photo-realistic Terrain Modeling and Visualization for Mars Exploration Rover Science Operations
}

\author{
Laurence Edwards, \\ Michael Sims \\ NASA Ames Research Center \\ Moffett Field, CA, USA \\ \{Laurence.J.Edwards, Michael.H.Sims\} \\ @nasa.gov
}

\author{
Clayton Kunz, David Lees \\ QSS Group, Inc. at \\ NASA Ames Research Center \\ Moffett Field, CA, USA \\ \{ckunz, lees\}@arc.nasa.gov
}

\author{
Judd Bowman \\ Massachusetts Institute of \\ Technology \\ Cambridge, MA, USA \\ jdbowman@mit.edu
}

\begin{abstract}
Modern NASA planetary exploration missions employ complex systems of hardware and software managed by large teams of.engineers and scientists in order to study remote environments. The most complex and successful of these recent projects is the Mars Exploration Rover mission. The Computational Sciences Division at NASA Ames Research Center delivered a $3 D$ visualization program, Viz, to the MER mission that provides an immersive, interactive environment for science analysis of the remote planetary surface. In addition, Ames provided the Athena Science Team with high-quality terrain reconstructions generated with the Ames Stereopipeline. The on-site support team for these software systems responded to unanticipated opportunities to generate $3 D$ terrain models during the primary MER mission. This paper describes Viz, the Stereo-pipeline, and the experiences of the on-site team supporting the scientists at JPL during the primary MER mission.
\end{abstract}

Keywords: Visualization, virtual reality, software architectures, simulation, stereo correlation, computer vision, surface reconstruction.

\section{Introduction}

The Mars Exploration Rovers (MER) mission is the first to have deployed truly mobile robotic science platforms on the surface of Mars. The two MER rovers, Spirit and Opportunity, have spent over one year navigating kilometers of distance and acquiring tens of gigabytes of image and spectral data [1]. This represents an orders of magnitude increase in mobility and data acquisition relative to previous Mars surface missions. To handle the complexity of managing these rovers, many disparate software tools that could not be fully tested prior to operations, were required to function cohesively in a mission critical environment. Additionally, diverse groups of people, in fields ranging from geology to software engineering to network communications, were required to work effectively in an organization that encompassed much more than any one individual's area of expertise. The challenge these circumstances created and the ultimate success that the MER mission experienced demonstrate the need for, and rewards of, employing a complex "system of systems" in modern planetary exploration.

Mars Exploration is a high priority for NASA. In the coming years even more ambitious surface missions, such as the Mars Science Laboratory, will be attempted and possibly culrainate in human exploration of the planet. As such, it is important for the participants in the current generation of robotic exploration to document and analyze their experiences dealing with complex interdisciplinary systems in order to better prepare for these future missions.

In this paper, we describe the visualization and surface reconstruction software provided to the MER mission and Athena Science Team by the Autonomous Systems and Robotics (ASR) area at NASA Ames Research Center (ARC), as well as the experiences of the on-site team supporting the scientists using this software at the Jet Propulsion Laboratory (JPL) during the primary MER mission.

\subsection{Background}

The ASR area at NASA ARC was responsible for delivering a science operations software element to the MER mission - a 3D visualization program named Viz. Designed to help scientists understand a wealth of data and provide enhanced situational awareness for science activities, Viz provided mission scientists with an easy to use, immersive, interactive $3 \mathrm{D}$ visualization environment. In addition to the Viz environment, Ames provided the Athena Science Team with high-quality terrain reconstructions. This capability was delivered with the Ames. "Stereo-pipeline," a software tool that generates dense 3D meshes from stereo image pairs. The ARC Viz support team assisted Viz users and, in particular, focused on responding to unforeseen opportunities to generate 3D data products - for example, Microscopic Imager (MI) data was used to create finely detailed rock surface models, and a mix of orbital and descent imagery was used to create digital elevation models of the area surrounding the MERA landing site.

NASA ARC has been involved in the research and development of immersive $3 \mathrm{D}$ visualization and surface reconstruction techniques since the early 1990s [2]. In particular, the ASR (formerly the Autonomy and Robotics Area) has focused on applying these technologies in the context of planetary exploration missions for science understanding and situational awareness. In the mid-1990s, NASA ARC developed the Ames Stereo-pipeline surface reconstruction software and the "MarsMap" visualization environment and provided these tools as a technology 
demonstration to the Mars Pathfinder (MPF) mission. The Ames Stereo-pipeline implemented a 3D-from-stereo capability for automatically producing accurate, highresolution, texture-mapped, 3D terrain models at downlink data rates. MarsMap could display the terrain models generated by the Stereo-pipeline in an immersive, interactive environment and provided science measurement and analysis tools [3].

The value of such immersive visualization aids for situational awareness was quickly demonstrated as scientists planned operations for MPF's Sojourner rover. Navigation hazards that were not visible in the 2D imagery or would have required manual photogrammetry to determine, were readily apparent in the MarsMap environment. In addition, MarsMap's measurement tools and annotation capabilities helped scientists determine distributions of rock sizes and wind streak directions - clues to the processes at work in an area.

Although quite effective, MarsMap was developed specifically for the MPF mission and was not architected for adaptability to other missions or applications. Leveraging the experience with MarsMap, ARC developed Viz for release to the Mars Polar Lander (MPL) mission as a next generation visualization environment with adaptability and extensibility as key architectural considerations. The concept of a "visualization server" was introduced, separating core 3D visualization functionality from application or mission specific capabilities and user interface (UI) components.

Also for MPL, a faster more robust version of the Ames Stereo-pipeline correlator was developed and integrated with JPL image rectification and camera calibration software. The integrated surface reconstruction software (the "James" pipeline) formed part of the mission's Ground Data Systems (GDS) downlink processing pipeline. Unfortunately, the mission was cut short due to a spacecraft fault during landing, and as a result Viz and the James pipeline only saw use during the mission in pre-landing operational readiness tests.

For the MER mission following MPL, Viz was provided as a Class B (mission enhancement) science tool. Although the Ames Stereo-pipeline only saw incremental changes, the Viz user interface was re-implemented for improved mobility support. In addition, the Viz architecture was extended to provide better support for a seamless user experience, additional simulation tools were integrated, and cross-platform portability was improved. Viz and the Ames Stereo-pipeline are described in some detail in the following section as they were deployed for MER.

In addition to planetary exploration missions, Viz and the Ames Stereo-pipeline have been used in several "FDO" and "K9" rover field tests between 1999 and 2004 [4][5]. Viz and the Ames Stereo-pipeline are also core components of ARC's Single Cycle Instrument Placement (SCIP) technology development program [6].

\section{$2 \mathrm{Viz}$}

Viz is a cross-platform, modular, extensible, visualization software package that allows users to interactively manipulate and investigate simulated 3D environments. The $\mathrm{Viz}$ environment was designed to support simple quantitative and qualitative scientific analysis of the remote planetary surface, as well as conceptual planning of science operations. Viz supports hardware stereo viewing capabilities utilizing LCD shutter glasses, and provides a variety of surface interrogation tools, lighting and rover simulation capabilities, and visual feedback cues to enhance the scientists understanding of surface topography. A web interface, the "Viz Browser," provides the Athena Science Team with easy access to Viz and available 3D model data. Viz implements a network based communication layèr providing an inter-process component interface, allowing integration of application specific tools that would be difficult to implement at the binary level due to differing implementation languages or incompatible architectures. In addition, the network interface provides an infrastructure for distributed applications. For MER, extensibility was further enhanced through the addition of a binary plug-in interface. This provides improved efficiency for inter-component communication and better support for tight user-transparent integration of components.

The separation between visualization server and application UT inherent in the foundational Viz architecture was leveraged for MER development, and the MPL UI was completely replaced with a MER relevant UT (see Figure 1) that has support for mobility: multiple sites and reference frames, multiple 3D view windows, and rover simulation. 

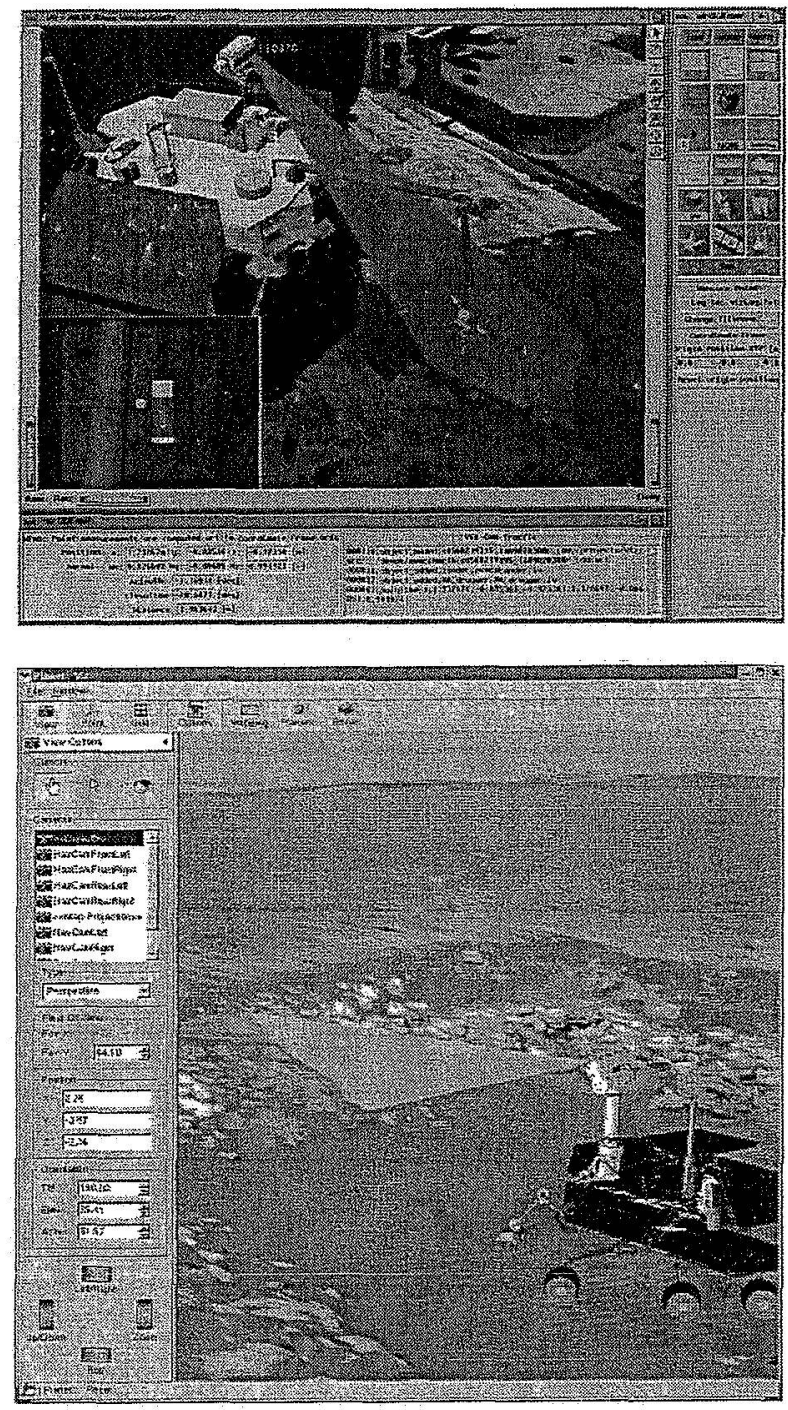

Figure 1. Top: the MPL Viz user interface. Bottom: the MER Viz user interface.

In addition to user interface changes, file input/output functionality had to be adapted to handle MER data products from the Athena payload and the MER platform. As a primary requirement, 3D model file formats defined by JPL GDS teams had to be supported in addition to the VRML file format generated by the Ames' Stereo-pipeline. This work included adaptation to both geometry and image file format specifications. Input of annotations (markers for science targets) from JPL's Science Activity Planner (SAP) GDS tool was also implemented, and an articulated 3D model of the MER rover was adapted for use within the Viz environment.

NASA ARC provided a number of software tools and capabilities to the MER mission, and the Viz team worked closely with other ARC teams to leverage synergies where possible and coordinate support. Viz plug-ins were developed to obtain data from ARC's Collaborative Information Portal (CIP) database infrastructure, and to allow Viz to work as a client within ARC's "MERBoard" electronic whiteboard environment. We also worked closely with the ARC Microscopic Imager (MI) Toolkit team during the mission support phase to provide scientists with a single access point for data products generated by the two teams.

As delivered to the MER mission, Viz implemented the following functionality:

- Interactive 3D fly-through

- Stereo view (hardware and anaglyph)

- Interactive time-of-day analysis: shadow simulation, sun and planetary body vectors

- Science measurement: location, distance, surface area, etc.

- Situational awareness: rover pose and viewpoint simulation

- Site understanding: terrain elevation profiles, color coded elevation and slope maps

- Rendering effects (fog, backgrounds, etc.)

- Compatibility with JPL generated GDS data products

- Display of SAP targets

- Compatibility with ARC's CIP and MERBoard

From a user's standpoint the primary new Viz functionalities implemented for MER were: multiple site support, multiple 3D views, rover simulation, and shadow simulation.

\subsection{Software Architecture}

$\mathrm{Viz}$ is implemented in the $\mathrm{C}++$ programming language. All toolkits or libraries used were either developed at $\mathrm{ARC}$, have freely available source code, or are Open Source. The two primary toolkits used in Viz are OpenInventor from SGI [7], for basic 3D scene graph functionality and management, and Trolltech's Qt crossplatform Open Source application framework [8]. Openlnventor was originally developed commercially by SGI, but was open-sourced in 2001.

Although originally selected as a UI toolkit, Qt has broad functionality and is now used throughout Viz. The Qt framework was the critical toolkit that enabled crossplatform support. Little work has to be done to deliver a version of $\mathrm{Viz}$ on a wide range of platforms. Although the primary MER delivery platform is Linux, we have at various times released Windows, Irix, and Mac OS X versions of Viz. 
The base functionality of Viz can be divided into four components: 1) binary plug-in component coordination, 2) inter-application communication, 3) internal 3D database management, and 4) user interface. Figure 2 is a schematic representation of this architecture. The signal-slot mechanism of the Qt framework is utilized to provide a message-passing infrastructure to connect binary plug-in components in a publish-subscribe model. Each binary plug-in component is a dynamically linked library and can initiate its own execution thread. The four base components are binary plug-in components themselves, and a discussion of each of the base components follows.

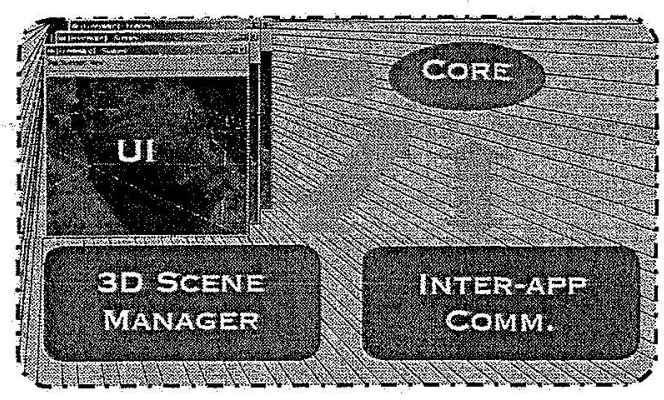

Figure 2. The Viz architecture. The system's functionality is divided into four base components.

\subsubsection{Plug-in component coordination}

The coordination between all the binary plug-in components occurs in the "Core" component. This component determines the run-time configuration (i.e., which components to load), loads all other components, and establishes the message-passing links.

Since components are self-contained and communicate through a common message infrastructure managed by the Core component, additional functionality for specific application specific tasks can be implemented in separate components and selectively loaded at runtime. These additional plug-in components operate transparently with the base system providing an extensible integrated feature set.

\subsubsection{Inter-application Communication}

Initially the primary means of inter-component coordination and communication, the inter-application communication component is now simply another binary plug-in component that acts as au intemediary between the Core component and external network components.

This component implements a socket-based communications layer using the External Data Representation Standard (XDR) protocol. XDR is an open standard [9] first used in the MPL version of Viz. Messages are asynchronously converted between the internal Viz format and the XDR protocol, enabling external processes, whether local or remote, to function for most purposes as if they were binary plug-in components.

\subsubsection{Internal 3D Database Management}

The OpenInventor high-level graphics library is used for rendering, modifying, and constructing the internal $3 \mathrm{D}$ scene graph database in the 3D database management component. OpenInventor was also used in the MPL version of $\mathrm{Viz}$, and the functionality implemented in this component along with the inter-application communication component and the UI component formed the conceptual basis for the original "visualization server".

This component supports loading 3D model data files conforming to the following specifications into the $3 \mathrm{D}$ environment:

- JPL's initial VISTA specification

- JPL's initial ASD specification

- Virtual Reality Modeling Language (VRML) version 1.0

- OpenInventor version 2.1 file format

- JPL's ITSA file format used with WITS for FIDO

Input files in the VRML and OpenInventor formats may utilize auxiliary image files that define 3D model textures. Image files in JPEG, RGB, GIF, and Planetary Data System (PDS) formats are supported. In addition to 3D file formats and image formats, Viz supports import of target files conforming to the ARES RML uplink target file format.

Manipulation and interrogation of the internal 3D database is handled through a queue of requests from other components. For complex environments, the time to process a request may be large, so the queue is optimized to improve efficiency by compressing multiple requests into one action. For example, during animated sequences, a scene element such as the rover camera mast, may be requested to rotate in small increments. If messages arrive faster than they can be processed, they are automatically composed into a single request representing the net effect of the rotation.

\subsubsection{User Interface}

The Qt graphical user interface classes are utilized to automatically provide a native look and feel for each supported platform. Two custom window classes are employed to logically divide functionality: 1) user initiated management of the internal 3D database is accomplished through a dedicated "main" window, and 2) multiple, interactive renderings of the environment are presented through "viewer" windows. In each window class, a docking toolbar paradigm allows access to functionality, with dialog windows for detailed tasks. As a user interacts with the simulated environment, action requests are sent to the internal $3 \mathrm{D}$ database management component for 
execution. The response is received and displayed by the user interface component.

\subsubsection{Application Specific Components}

For the MER mission, in addition to the base components, four application-specific binary plug-ins were developed: 1) A kinematic simulation component based on the VirtualRobot program developed at the Ecole Polytechnique Federale Lausanne (EPFL) and NASA ARC [10]. This component provides a forward and inverse kinematics simulation capability, 2) A solar system ephemeris component. This is utilized for time of day lighting simulation and planetary body location functionality, and utilizes the JPL SPICE libraries, 3) a CIP component allowing access to data in the CIP database, and 4) a MERBoard component implementing the MERBoard client interface.

A network component was also developed that could parse MER engineering telemetry files, allowing playback of executed rover command sequences in the Viz environment.

\section{Surface Reconstruction}

The Ames Stereo-pipeline incorporates a fast image correlation algorithm for calculating positional disparities between corresponding points in left and right images of a stereo pair. From the image disparities, 3D points are calculated using the known camera geometry, and these points are used to create a $3 \mathrm{D}$ triangle mesh. In addition, various pre- and post-processing modules in the pipeline can be invoked to condition the data for improved performance when correlation is difficult, or to optimize the generated mesh.

As with MPL, image rectification and distortion removal was primarily achieved with JPL's GDS image processing tools. However, it was still necessary to adapt Ames' stereo pipeline to accept the PDS image file format used by the mission, and to read camera model information from image headers. For MER, the camera geometry and lens distortion information is specified using variants of the "CAHV" model [11].

The Cornell Pancam team provided accurate radiometric calibration information for Pancam images in derived image product headers. The Ames Stereo-pipeline was adapted to use this information to correct for image brightness differences due to varying lighting conditions over the duration of an image panorama acquisition. The Pancam team also provided initial color adjustment information that allowed us to generate near true color 3D model textures from sets of monochrome images acquired with the Pancams color filters.

\subsection{Non-standard 3D. Data Products}

In addition to standard $3 \mathrm{D}$ data products generated from matched left-right stereo image pairs, $3 \mathrm{D}$ models were also generated from individual overlapping images. In particular, the Pancams have a somewhat narrow field of view, which prevents them from imaging targets close to the rover in left and right cameras simultaneously. However, scientists attempted to overcome this limitation by moving the Pancam Mast Assembly (PMA) between two individual image acquisitions to achieve overlap. To build 3-D models from these somewhat unorthodox stereo pairs, the assumption was that the PMA kinematics were well enough understood that camera position telemetry was essentially exact. In practice, the only artifact evident was a minimal amount of warping near the edges. The scale of objects reconstructed in this way is also not as precise as with calibrated Pancam stereo cameras pairs. The same technique was used to reconstruct 3-D models from pairs of microscopic images, where camera repositioning is achieved with motion of the IDD. Finally, 3D models from overlapping Mars Global Surveyor (MGS) and MER descent imagery were generated. Scale was roughly calculated from knowledge of the orbiters and altitude and a planarity assumption

\section{MER Science Support Experiences}

Members of the Viz, MERBoard, CIP, and MI Toolkit teams were on-site at JPL during the first 90 days of the MER mission. The Viz and MI Toolkit teams in particular worked closely together due to commonalities in data processing tools and the complimentary nature of the data products generated by the two teams. Our presence at JPL proved to be critical to our successful support of the MER science team. In the following we describe the joint support provided by the Viz and MI Toolkit teams, and lessons learned from the experience.

\subsection{Science Support Tasks}

Technologists' naturally want to tune any tools they develop to address the most typical challenges with which they are confronted. For efficient production of good quality visualizations, the obvious approach was to ensure that the stereo reconstruction processes we used behaved well on the most common sorts of terrain. Unfortunately, the scientists' interests often were in direct conflict with this strategy. Their attention focused most immediately on the closest atypical terrain features, as these are the things that were the most interesting to them. As a result, one of the greatest assets we had as a team was the ability to adapt our tools and methods "on the fly" to meet the varied needs of the science team.

\subsubsection{Microscopic Imager Data Analysis}

One of our more successful efforts during the mission was to support the analysis of cross bedding (layering) in the rocks at the Opportunity landing site. Images of the 
rocks taken with the Pancam sometimes did not provide sufficient resolution for in depth science analysis. The microscopic imager (MI) offered much better resolution of the rock surface. However, the MI has an extremely narrow field of view (only about $2 \mathrm{~mm}$ by $5 \mathrm{~mm}$ ), and has only a single camera, which makes stereo reconstruction problematic.

Our team worked with the mission scientists to create and register a mosaic of approximately $120 \mathrm{MI}$ images and we adapted the Ames Stereo-pipeline to reconstruct 3-D models of the rock's surface based on knowledge of the movement of the rover's Instrument Deployment Device (IDD) between successive MI images. The models constructed using these opportunistically developed processes allowed the mission scientists to take measurements of the geometry of the rocks at the landing site to support their analysis of cross-bedding,

Another analysis task we performed while on site at $J P L$, was the creation of models where $M I$ and Pancam stereo data were "fused" and geometrically registered, so that scientists were able to see (for example) the overall shape of a rock, with a highly detailed patch of MI data correctly registered at an area of interest (see Figure 3). As with the MI mosaic described above, we were able to adapt our tools and methods to an unanticipated requirement rapidly enough to provide additional analytical support to the scientists during the mission.

\subsubsection{Terrain Visualization Support}

The creation of accurate, smooth terrain models from stereo imagery is part art and part science. While the JPL GDS image processing tools did construct stereo models from a portion of the stereo image data available, they were not optimized for visual quality or to support specific science analysis - the data processing volume was simply too high, and rover operational concerns were paramount. Since our team was focused solely on science activities and analysis, we were able to devote our time to working with the scientists to identify specific areas of interest and then optimize the 3-D models of those areas.

As described above, Viz provides for the viewing of 3-D terrain models in stereo with hardware support for LCD shutter glasses. This proved to be a valuable tool for the science team to gain contextual awareness of the rover's surroundings, as terrain features that are not obvious in 2-D projections are sometimes very prominent when viewed in 3-D. The geology and long term planning theme groups of the science team in particular made frequent use of stereo viewing capabilities.

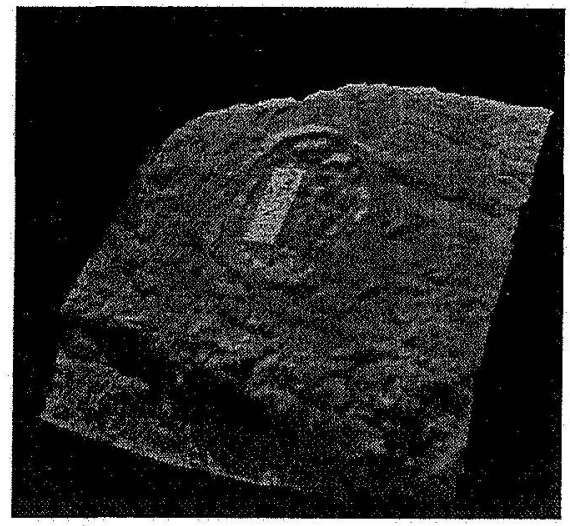

Figure 3. A 3-D model of the rock "King 3" at the MER-B landing site. The false color model was created from Pancam imagery, with a 3-D MI model superimposed over an area that was surfaced with the RAT.

\subsection{Data Browsing/Management}

"Round-trip data tracking" was not fully supported by the official mission software tools, because of limited time and resources prior to the landing of the rovers. Because of the complex workflow that an activity plan followed before being delivered to the rovers, and the high volume of data returned from the rovers, it was a challenge to correlate the returned data with the plan that had initiated its collection. Absent conferral with the science team member or group that had created a particular plan, it was very difficult to "reverse-engineer" the purpose behind a given collection of returned images.

We developed two interfaces to help organize data access. The "Viz Browser" cataloged the 3-D models our team produced, and connected them to the original 2-D images used to generate the 3-D models. Similarly, the "Microscopic Image Browser" helped to organize the data returned from the $M I$ and correlate raw images with derived data products. Both interfaces were accessed through a common "Ames Browser" web interface.

\subsection{Value of Direct Contact with Science Team}

Ultimately, there were two major "lessons learned" from our MER experiences, both of which argue strongly for the value of having technical teams present during the mission. First, the tight schedules and odd hours (synchronized to Martian rather than Earth days) made external collaboration all but impossible. It would have been very difficult to coordinate with members of the science team if we had not been there working under the same schedule and deadline pressures. Second, the extreme scheduling demands of mission operations produced a very reasonable "expedience driven" mindset amongst the scientists. Whenever possible, the scientists preferred to use their own computers and familiar tools such as Photoshop and PowerPoint. The scientists overriding priority was to get their tasks completed in the fastest most direct way possible. What this meant for us was that the 
real resource we provided was our presence at JPL and our willingness and ability to adapt our processes and tools to support a variety of science analysis tasks, not the software tools we developed in and of themselves. This was true for the analytical tools and data products we provided, and even to a certain degree for mission critical GDS tools.

The demanding schedule and huge volume of tasks required for mission operations means that it is probably not realistic to expect scientists to develop expertise in all of the software tools available on a typical mission, due to the wide variety. Conversely, having a technical team available and ready to support a variety of science tasks with a set of tools familiar to them, proved to be very productive and valuable.

\section{Conclusions}

As both a standalone application and networked graphics server, Viz delivers a high degree of flexibility to support the needs of NASA's evolving robotic planetary exploration program. During the MER mission, Viz and the Ames Stereo-pipeline were able to be adapted by the on-site support team to provide scientists with valuable information that was not anticipated before the landed operations. With the trend of increasing complexity and higher expectations for NASA planetary exploration missions, the need for unanticipated analysis capabilities is likely to continue, and even increase. The experiences of this team suggest that, in order to maximize the return from these missions, it is critical to incorporate and utilize system elements that can quickly and easily change to meet unforeseen needs.

\section{Acknowledgements}

Many have contributed to the work presented in this paper. In particular we would like to thank: MER PI Steve Squyres, MER Deputy PI Ray Arvidson, and MER Co-Is Jim Bell and Ken Herkenhoff for their support; ARC team members Beau Crawford, Randy Sargent, Anne Wright, Matt Deans, and Mike Wagner; at JPL, the SAP development team, the rover engineering team, and Eric Dejong and the MIPL teân. Finally, we thank Dañ Maas from Maas Digital for providing the original MER rover model.

\section{References}

[1] Bell, J. F., et al, "Pamcam Multispectral Imaging Results from the Opportunity Rover at Meridiani Planum", Science, Vol. 306, Pg. 1703, December 3, 2004.

[2] Nguyen, L., et al., "Virtual Reality Interfaces For Visualization and Control of Remote Vehicles", Autonomous Robots, 11(1) 2001.
[3] Stoker, C., Zbinden, E., et al., "Analyzing Pathfinder Data Using Virtual Reality and Superresolved Imaging", Journal of Geophysical Research, vol. 104, no. E4, pp. 8889-8906, April 25, 1999.

[4] Bresina, John L. et al., "K9 Operation in May '00 Dual-Rover Field Experiment", iSAIRAS Proceedings, June 2001.

[5] Carol R. Stoker, et al., "Two Dogs, New Tricks: Field Trials with the K9 and FIDO Rovers at Black Rock Summit, Nevada", Journal of Geophysical Research, special issue on Black Rock Summit Field Test. 2002.

[6] Pedersen, L., et al, "Mission Planning and Target Tracking for Autonomous Instrument Placement", Proceedings of the 2005 IEEE Aerospace Conference, Big Sky, MT USA, March 2005.

\section{[7] http://oss.sgi.com/projects/inventor/}

\section{[8] http://www.trolltech.com/products/qt/}

\section{[9] http://wrww.faqs.org/rfesirfel 832.html}

[10] Flueckiger, L., "A Robot Interface Using Virtual Reality and Automatic Kinematics Generator", Proceedings of the $29^{\text {th }}$ International Symposium on Robotics (ISR 98), Birmingham, U.K., April 1998, pp. 123-126.

[11] Y. Yakimovsky and R. Cunningham, "A System for Extracting Three-Dimensional Measurements from a Stereo Pair of TV Cameras," Computer Graphics and Image Processing, No. 7, pp. 195-210, 1978. 